\title{
Experimental analysis for the effect of dynamic capillarity on stress transformation in porous silicon
}

\author{
Wei Qiu, Yi-Lan Kang, ${ }^{\text {a) }}$ and Qiu Li \\ Department of Mechanics, Tianjin University, Tianjin 300072, People's Republic of China \\ Zhen-Kun Lei \\ Department of Engineering Mechanics, Dalian University of Technology, Dalian 116024, People's Republic \\ of China \\ Qing-Hua Qin \\ Department of Engineering, Australian National University, Canberra, Australian Capital Territory 0200, \\ Australia
}

(Received 26 October 2007; accepted 8 January 2008; published online 30 January 2008)

\begin{abstract}
The evolution of real-time stress in porous silicon (PS) during drying is investigated using micro-Raman spectroscopy. The results show that the PS sample underwent non-negligible stress when immersed in liquid and suffered a stress impulsion during drying. Such nonlinear transformation and nonhomogeneneous distribution of stress are regarded as the coupling effects of several physical phenomena attributable to the intricate topological structure of PS. The effect of dynamic capillarity can induce microcracks and even collapse in PS structures during manufacture and storage. (C) 2008 American Institute of Physics. [DOI: 10.1063/1.2838716]
\end{abstract}

Because of its special properties including large specific surface area, high reactivity, diversiform topological structure, and photo-electroluminescence, porous silicon has been widely used in recent years in microelectromechanical systems, ${ }^{1}$ optoelectronic/microelectronic devices, ${ }^{2}$ and clinical medicine/pathology biosensors. ${ }^{3}$ Nevertheless, collapses and cracks often happen when porous silicon (PS) either in service or during manufacture and storage processes. Previous works on the mechanical behavior of PS structures mostly focused on the failure caused by intrinsic residual stress ${ }^{4-6}$ and a few of them investigated the damage phenomena relevant to capillarity. ${ }^{7-11}$ However, there is still a lack of elaborate experiment investigating the effect of dynamic capillarity on the stress transformation in porous structure such as PS.

In this letter, a dynamical capillarity experiment is preformed to analyze the real-time stress transformation during PS drying by applying micro-Raman spectroscopy. Using the experimental results, the mechanical phenomena and their physical sources during dynamical capillarity in PS are studied. Finally, the failure mechanisms by capillarity are discussed.

Raman scattering originates from the interaction and energy exchange of an incident light with phonons in the sample. $^{12}$ When the sample undergoes an intrinsic or mechanical stress, its Raman peak shifts from its original position $w_{0}$ to $w$. The amount of this shift is linearly proportional to the equivalent stress $\sigma$ as Eq. (1) by applying Hooke's law for elasticity and the "secular equation,",

$$
\begin{aligned}
\Delta w & =w-w_{0} \approx \frac{\lambda}{2 w_{0}}=\frac{1}{2 w_{0}}\left[p S_{12}+q\left(S_{11}+S_{12}\right)\right] \sigma \\
& =\operatorname{RSS}^{-1} \sigma,
\end{aligned}
$$

where $p$ and $q$ are phonon deformation potentials, $S_{\mathrm{ij}}$ are elastic compliance tensor elements, and RSS is named as the

\footnotetext{
${ }^{a)}$ Electronic mail: tju_ylkang@yahoo.com.cn.
}

\section{Raman shift to stress coefficient.}

In this experiment, PS samples were fabricated by electrochemical etching on 2 in. (100) silicon wafers $\left(p^{+}\right.$type, $0.01-0.02 \Omega \mathrm{cm}$ ) with a $1: 1 \mathrm{HF} /$ ethanol solution. ${ }^{15}$ The PS layer was about $30 \mu \mathrm{m}$ in thickness and its porosity was estimated as $60 \%$ by means of usual gravimetric method. A cross-sectional photograph [Fig. 1(a)] of the sample obtained using a field-emission scanning electron microscope (FESEM) shows that the topological structure roughly resembles a bushy flourishing forest, as Fig. 1(b) illustrates. The pillars are nearly parallel with each other and vertical to the plane of PS film. The diameters of both pores and pillars are several tens of nanometers, about one magnitude larger than the size of grains and subpores. All these measurements are consistent with those of the samples in Refs. 16 and 17. Besides, as for the sample (with $60 \%$ porosity) used in current work, its RSS is $38.19 \mathrm{MPa} / \mathrm{cm}^{-1}$. $^{14}$

The experiments in this work applied a Ranishaw RM2000 microscopic confocal Raman system with a $514 \mathrm{~nm}$ $\mathrm{Ar}^{+}$laser exciting light whose output was regulated to $1 \%$ $(0.23 \mathrm{~mW})$ to avoid the laser-heating effect. A $50 \times$ Olympus objective was selected; hence, the spot size of incident laser was about $2 \mu \mathrm{m}$ in diameter.
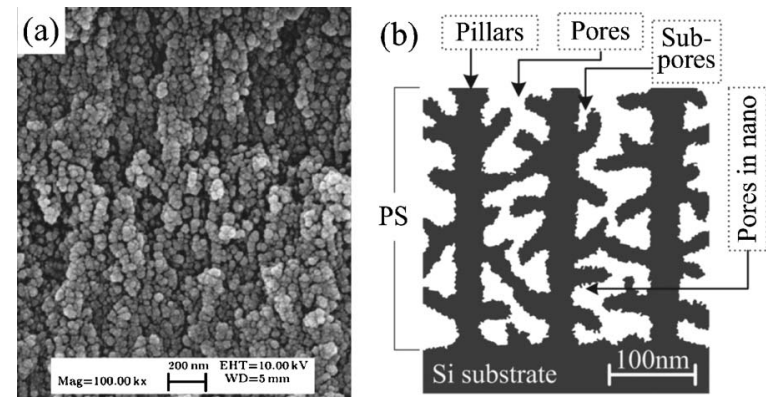

FIG. 1. (a) Cross-sectional photograph of PS sample with $60 \%$ porosity by FE-SEM. (b) Diagrammatic sketch of PS topological structure. 


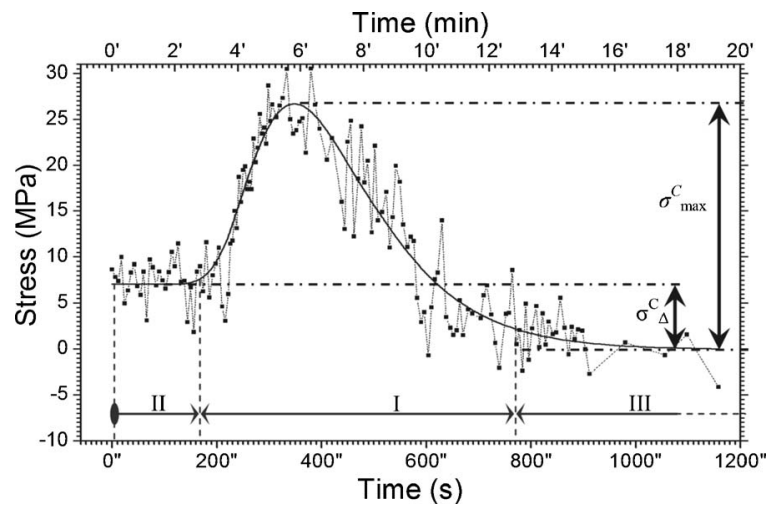

FIG. 2. Experimental result of planar stress transformation on PS surface during dynastic capillarity.

The dynamic capillarity test was performed at room temperature in the following way. Firstly, a PS specimen was placed in a small culture dish, then the dish was set on the object stage of Raman microscope, and the incident spot was focused on the PS surface. After that, ethanol was infused into the culture dish until the liquid submerged the sample by about $0.1 \mathrm{~mm}$. The sampling and interval times were initialized as 1 and $5 \mathrm{~s}$, respectively. Then, acquisition of Raman data began. When the fluid in the culture dish had almost volatilized, the sampling interval time was gradually extended from $5 \mathrm{~s}$ to $1 \mathrm{~min}$ until the sample becomes absolutely dry. The whole process lasted $\sim 20 \mathrm{~min}$.

All the Raman spectra were fitted by Lorentzian distribution function to obtain their Raman shifts and then substituted Eq. (1) (taking the statistic mean of Raman shifts when the PS in the dry state as $w_{0}$ ). Hence, the real-time stress transformation in the PS during dynamic capillarity was achieved and is shown in Fig. 2, where the scattered square spots are the stresses taken from experimental data and the solid line is the time-stress curve obtained by fitting experimental data from discrete sampling moments.

The Raman-test results in Fig. 2 show that the stress in the PS film induced by capillarity changes nonlinearly with time. It is evident that the time-stress curve is varying acutely during the middle segment and approximately stable in both the beginning and the end segments. Hence, the stress transformation can be divided roughly into three stages, named as stages I, II, and III, respectively, (dashed line in Fig. 2). The detailed mechanical phenomena and their possible physical sources in each stage are now analyzed.

In stage I of the test, the capillarity plays an important role in the stress transformation with the liquid level traversing the PS layer [Fig. 3(a)]. In a random pillar [shown in Fig. $3(\mathrm{~b})$ ], the value of contact angle between $\mathrm{Si}$ and ethanol is

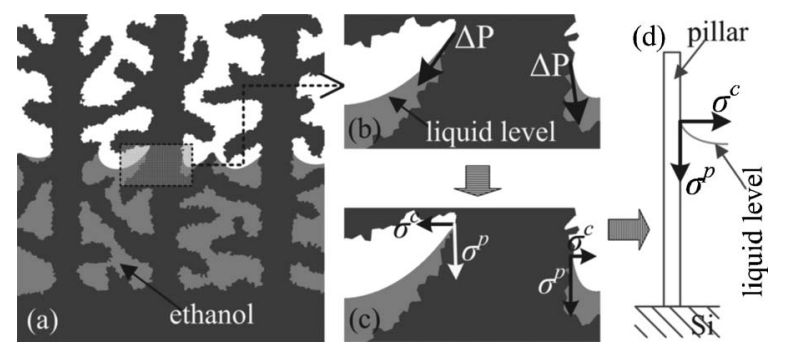

FIG. 3. Diagrammatic sketches of (a) PS in wet, (b) adsorption force on random pillar, (c) stress resolution of (b), and (d) $\sigma^{C}$ decreases with liquid level descending. PS in manufacture and storage become clear. As for the me-
level descending.
Downloaded 23 Mar 2009 to 150.203.161.6. Redistribution subject to AlP license or copyright; see http://apl.aip.org/apl/copyright.jsp uniform but the air-liquid-solid triphase interfaces lie in different directions at different points of the pillar as it is flexuous. Hence, the adsorption force $\Delta P$ on the pillar is asymmetric. Through stress resolution, $\Delta P$ is equivalent to a tensile stress $\sigma^{t}$ and a compressive stress $\sigma^{p}$, parallel with and vertical to the PS film, respectively, as shown in Fig. $3(\mathrm{c})$. Both $\sigma^{t}$ and $\sigma^{p}$ are inharmonic and unsteady because of the rough surface of the pillar. Acting as external loads, they induce a nonlinearly varying intrinsic stress in PS, namely, $\sigma^{C}$, which is measured by Raman experiment in this work (Fig. 2). Such a nonhomogeneneous stress may cause the PS to fail during its manufacture and storage discussed at subsequent paragraphs.

The Raman data shown in Fig. 2 indicate that, as time progresses, the stress in PS increases quickly to the maximum $\sigma_{\max }^{C}$, about $26.7 \mathrm{MPa}$, and then decreases gradually. Such a stress-impulsion phenomenon may mainly result from the alternations of two kernel factors: the one is the area of air-liquid-solid triphase interfaces (ATI for short) that is linearly proportional to the magnitude of capillary force, and the other is the location of the liquid level where $\sigma^{t}$ and $\sigma^{p}$ apply. With the liquid level continually descending, some taller pillars are first exposed to the air and liquid bridges form between them. As more and more pillars/subpillars emerge from the fluid, the ATI increases quickly to its plateau, leading $\sigma^{C}$ to rise rapidly at the forepart of stage I. At the same time, by simplifying the pillar in forestlike porous structure as a cantilever beam [illustrated in Fig. 3(d)], we can see that the capillary with different heights of liquid level induces different intrinsic stresses. Since the liquid level goes closer to the pillar root gradually, $\sigma^{t}$ plays a diminishing influence on the stress in PS.

In stage II (the beginning of the test), the PS sample is totally submersed by ethanol. It seems that the external loading condition remains steadily. However, it is noted that the PS sample is also subjected to a stress, $\sigma_{\Delta}^{C}=7.1 \mathrm{MPa}$, due to the immersion. In contrast, there is no such stress when the sample is in dry air. The mechanism of $\sigma_{\Delta}^{C}$ can be explained as follows. When the whole sample is immerged in liquid, the pores inside the PS are not fully filled, as described in the work of Moretti et al. ${ }^{18}$ This phenomenon perhaps owes to the nanoscale effect of the specimen since we can see from Fig. 1 that the trunk pores and pillars are all in the magnitude of $10-100 \mathrm{~nm}$, whereas the grain and subpores are several nanometers only. It is non-negligible that capillarity may occur when the gas and liquid phases coexist in the pores with nanoscale. Besides, several nonclassical stresses and physical phenomena, including Van de Waals force, ${ }^{11}$ oxidation stress, and Casimir effect, ${ }^{19}$ may contribute to the stress of PS when it is immerged in alcohol.

The test of dynamic capillarity enters stage III when the liquid level moves below the PS/Si interface. Nonetheless, the capillary effect in the PS does not vanish immediately. And even when the test approaches the boundary of stages I/III, the stress decreases with an obvious deceleration. The existence of stress in the PS after the pillars are exposed to the air is probably attributable to the fact that there is some residual ethanol remaining in the poses, maybe partly due to the capillary condensation ${ }^{20}$ happened in nanopores, which induces some capillarity and gasifies with a lingering speed.

Based on the above analysis for the mechanism of stress transformation in PS during drying, the failure problems of 
chanical behavior of PS devices, most existing studies have attributed the collapse, cracking, and spalling of PS to residual stresses, or rather, to intrinsic stresses owing to lattice mismatch ${ }^{4}$ and distortion, ${ }^{5}$ while capillarity and other processing stresses have always been neglected. Nevertheless, capillarity is essential for obtaining uniform high-quality PS during some key processing steps such as immersion, rinsing, and drying..$^{7-9}$ The capillarity is reversible if the sample $^{7}$ is free from structural failure or chemical denaturation. ${ }^{9}$ However, if any acute or repeated "wet-dry-wet" processes occur during its fabrication or even if it is stored in liquid for a long time, PS film will undergo the impulsion of nonlinear and inhomogeneous stress, as shown in Fig. 2, and may be torn into "islands" or peeled from the Si substrate during the dynamic capillarity discussed above. The failure mode of PS is relative to its topological structure. For the forestlike PS, as the cross linkages among pillars are far weaker than the interfaces between pillars and Si substrate [see Fig. 1(b)], the stress by capillarity is more likely to lead the PS to crack rather than to peel from the wafer, which actually happened in our PS preparation and also consistent with the failure phenomena in others works. ${ }^{10}$

In summary, the stress induced by capillarity is a primary "processing stress" during PS preparation and storage. This letter presents an experimental analysis, by applying micro-Raman spectroscopy, for the real-time stress transformation during the processing of PS from wet to dry. Experimental results show that the sample suffers a stress impulsion increasing quickly to a maximum and then decreasing gradually before vanishing. Moreover, the PS is subjected to non-negligible stress even though immersed in ethanol. These stress phenomena observed are likely due to the coupling effects of several mechanical and physical phenomena taking place inside the multidimensional and intricate topological structure of the PS, coincident with the liquid volatilizing. As the result, the inhomogeneous stress induced by dynamic capillarity during either rinsing, drying, or immersing may cause the porous silicon to crack and collapse, which can significantly affect the quality of PS during its manufacture and storage.

This work is funded by the National Natural Science Foundation of China (Contract Nos. 10732080 and 10502014). The authors would also like to acknowledge Professor Ming Hu and Dr. Zhen-Qian Fang for providing porous silicon samples.

${ }^{1}$ G. Kaltsas and A. G. Nassiopoulos, Microelectron. Eng. 35, 397 (1997).

${ }^{2}$ L. T. Canham, Appl. Phys. Lett. 57, 1046 (1990).

${ }^{3}$ V. S.-Y. Lin, K. Kianoush, K.-P. S. Dancil, M. J. Sailor, and M. R. Ghadiri, Science 278, 840 (1997).

${ }^{4}$ S. Manotas, F. Agullo-Rueda, J. D. Moreno, F. Ben-Hander, and J. M. Martınez-Duart, Appl. Phys. Lett. 75, 977 (1999).

${ }^{5}$ L. Pascual, R. J. Martín-Palma, A. R. Landa-Cánovas, P. Herrero, and J. M. Martínez-Duart, Appl. Phys. Lett. 87, 251921 (2005).

${ }^{6}$ H. S. Kim, E. C. Zouzounis, and Y. H. Xie, Appl. Phys. Lett. 80, 2287 (2002); Y. L. Kang, Y. Qiu, Z. K. Lei, and M. Hu, Opt. Lasers Eng. 43, 847 (2005); T. Unagami, J. Electrochem. Soc. 144, 1835 (1997); P. G. Abramof, A. F. Beloto, A. Y. Ueta, and N. G. Ferreira, J. Appl. Phys. 99, 024304 (2006).

${ }^{7}$ D. Bellet and G. Dolino, Phys. Rev. B 50, 17162 (1994).

${ }^{8}$ G. Amato, V. Bullara, N. Brunetto, and L. Boarino, Thin Solid Films 276, 204 (1996).

${ }^{9}$ V. Chamard, C. Pichat, and G. Dolino, Solid State Commun. 118, 135 (2001).

${ }^{10}$ M. D. Mason, D. J. Sirbuly, and S. K. Buratto, Thin Solid Films 406, 151 (2002).

${ }^{11}$ U. Grüning and A. Yelon, Thin Solid Films 255, 135 (1995).

${ }^{12}$ D. A. Long, Raman Spectroscopy (McGraw-Hill, New York, 1977).

${ }^{13}$ E. Anastassakis, A. Cantarero, and M. Cardona, Phys. Rev. B 41, 7529 (1990).

${ }^{14}$ Z. K. Lei, Y. L. Kang, H. Cen, and M. Hu, Chin. Phys. Lett. 23, 1625 (2006).

${ }^{15}$ B. Tian and M. Hu, Int. J. Inorg. Mater. 20, 545 (2005).

${ }^{16}$ K. Barla, G. Bomchil, R. Herino, J. C. Pfister, and J. Baruchel, J. Cryst. Growth 68, 721 (1984).

${ }^{17}$ X. G. Zhang, J. Electrochem. Soc. 151, 69 (2004); L. N. Aleksandrov and P. L. Novikov, Thin Solid Films 330, 102 (1998); R. J. Martín-Palma, L. Pascual, P. Herrero, and J. M. Martínez-Duart, Appl. Phys. Lett. 87, 211906 (2005).

${ }^{18}$ L. Moretti, L. De Stefano, and I. Rendina, J. Appl. Phys. 101, 024309 (2007).

${ }^{19}$ E. Buks and M. L. Roukes, Phys. Rev. B 63, 033402 (2001).

${ }^{20}$ K. B. Jinesh and J. W. M. Frenken, Phys. Rev. Lett. 96, 166103 (2006). 